\title{
Co-Operation as an Asymmetric Form of Human-Computer Creativity. Case: Peace Machine
}

\author{
Mika Hämäläinen \\ Department of Digital Humanities \\ University of Helsinki \\ mika.hamalainen@helsinki.fi
}

\author{
Timo Honkela \\ Department of Digital Humanities \\ University of Helsinki \\ timo.honkela@helsinki.fi
}

\begin{abstract}
This theoretical paper identifies a need for a definition of asymmetric co-creativity where creativity is expected from the computational agent but not from the human user. Our co-operative creativity framework takes into account that the computational agent has a message to convey in a co-operative fashion, which introduces a trade-off on how creative the computer can be. The requirements of co-operation are identified from an interdisciplinary point of view. We divide cooperative creativity in message creativity, contextual creativity and communicative creativity. Finally these notions are applied in the context of the Peace Machine system concept.
\end{abstract}

\section{Introduction}

When we say something in a language, we say it to communicate something. Every utterance we say has a meaning behind it, a message we want to convey to others. This is true not only in everyday conversation, but in any act of language use, no matter the medium, whether it was spoken, written, signed etc.

For computationally creative systems, exhibiting linguistic creativity, expressing a message is not a requirement. In fact, just generating a linguistic realization, a surface form, is challenging enough and is considered of a merit.

The situation becomes more difficult when mere surface generation, i.e. producing natural language without a message, is not enough. When a system has to generate a creative poem that expresses a complete message or has to make a meaning conveying contribution to a conversation. It is often the case that a computationally creative system is not fully aware of the meaning its creations convey, but rather rely on people to pour their understanding of the world into the creative artifact and perceive creativity in it.
In this paper, we focus on co-operative creativity with the focus on dialog systems. We are not greatly interested in purely generative dialog systems that serve more for chitchat. Instead, we focus on goal-oriented dialog systems that have a clear message they need to convey, such as a price or available times, and the role of computational creativity in encapsulating their message in a creative form.

Creative behavior consisting of a human and a computer is called co-creativity. In the following section, we start by discussing this notion and why it is insufficient for modelling our task. In the following sections, we take an interdisciplinary view on what co-operation means and formulate a creative framework based on these notions. Finally we show a more concrete way of using our framework by applying it on the Peace Machine concept.

In the field of computational creativity, working with a definition for creativity plays a crucial role in evaluation of a creative system (Jordanous, 2012; Alnajjar and Hämäläinen, 2018). While a myriad of more abstract level theories on computational creativity have been elaborated in the past (Colton, 2008; Wiggins, 2006; Colton et al., 2011), our work aims to develop a theoretical framework to a more concrete problem of creative dialog generation.

\section{Co-Creativity}

In this section, we describe some of the existing definitions of human computer co-creativity as the co-creativity paradigm is closest to our case.

Co-creativity can be divided into four categories as identified by Lubart (2005). The computer can act as a nanny to a person guiding and motivating him in the creative task, where as if the computer acts as a coach, it will more actively help the 
creative person to explore new ways of thinking by educating them about different creativity techniques. In a pen-pal scenario, the computer helps a creative individual in communicating ideas with others. Finally, the computer can be a colleague in which case humans and computers are in a creative dialogue taking turns in forming a creative artefact.

Davis (2013) identifies a gap between the AI research focusing on computational creativity and HCI (human-computer interaction) research focusing on creativity support tools. He argues that co-creativity can narrow this gap. Creativity is seen as an emergent phenomenon from the interactions of a human and a computer. The interactions are collaborative and both parties influence on each other.

In mixed initiative co-creativity (Yannakakis et al., 2014), both the computer and a human user take an active role in contributing to solving a creative problem, although, not necessarily to the same extent. This differs from turn-based collaboration between the two parties and from the computer being merely a supportive tool, as the both parties are actively creative.

In a recent study outlining evaluation of cocreativity (Karimi et al., 2018), the concept of cocreativity is defined as an interaction involving at least one AI agent and one human. They act based on the creative response of the other party and their own understanding of creativity.

The current definitions of co-creativity always expect the presence of human creativity in addition to computational creativity or computer assisted creativity. However, a co-operation setting does not require creativity at all, and if the computational agent is creative, it does not mean that there has to be human creativity present at the same time.

\section{Co-Operation}

Co-operative creativity requires the computer to exhibit creativity in its way of communication. However, creativity is not a requirement for the human user. Even though dialogue itself can be seen as an interplay between two or more parties forming an ephemeral creative artefact of its own, we want to clearly distinguish co-operative creativity from co-creativity. Therefore, we are not looking at dialogue as a creative artefact but rather how creativity can take place one-sidedly on the level of utterances.

\subsection{Communicative-Creative Trade off}

The purpose of a dialogue system, whether it is made for chitchat or to answer queries, is always to co-operate with a human. Co-operation can thus, in its simplest form, be contributing to the conversation in a meaningful way to keep the conversation on going.

The rules of conversation are governed by linguistic, cognitive and social mechanisms that have to be followed, and they set limitations for creativity. For instance, a dialogue system for booking movie tickets can deliver a very uncreative communicative answer stating just the name of the movie and its showtime or on the other extreme of creativity, answering by a riddle.

We argue that the co-operative nature of conversation, where creativity is only expected from the computer, not from the human, and where a certain communicative function has to be filled in accordance to higher level rules of conversation, has to balance in between creativity and predictability.

\subsection{Communication in Pragmatics}

The field of pragmatics has been studying meaning in its context for multiple decades. In this section, we will explain the key pragmatic theories in understanding conversation and meaning of utterances.

Grice (1975) famously defined four maxims for co-operative principle of communication: manner, quality, quantity and relevance. Through these maxims, we can identify linguistic rules that a machine should follow in order to be converse in a co-operative fashion.

The maxim of manner means that the communication is conducted in an orderly and unambiguous fashion. The maxim of quality refers to the truthfulness of the utterance. The speaker shall not say anything he believes to be false.

If there is just enough information communicated in an utterance, the maxim of quantity is followed. This means that both communicating too little or too much is against this maxim. The last maxim, namely that of relevance, requires the utterance to be contextually related and not off topic.

When it comes to the function of utterances, i.e. their relation to the surrounding world, we can use Searle's speech acts (Searle, 1969) (cf. Nonaka, 1994; Rus et al., 2012). According to this theory, 
all utterances are either representative, expressive, declarative, commissive or directive.

Representative and expressive are close to each other in a communicative function. The former states something factual about the surrounding reality outside of the speaker, where as the latter is a statement about the internal state, such as the emotion, of the speaker.

Directive speech acts are commands, i.e. their intention is to make someone else perform an action. Commissive speech acts have a similar function as they are promises, in their case the speaker is the one who is going to perform the action. Declarative speech acts are, by their definition, supposed to change the surrounding world. An example of such a speech act is sentencing someone guilty of a crime.

It is important to note that the surface form of an utterance does not dictate the speech act it is used to perform, but rather its contextualization plays an important role. For instance, a prayer is an expressive speech act even though on the surface it might seem as a directive speech act. This interplay between the context and the words themselves opens up a great potential for creativity.

\subsection{Socio-Cognitive Views}

In cognitive science, the concept of scripts (cf. Bower et al., 1979) can be used in a higher level to explain communication. In day-to-day life, our brains rely on heuristics when processing information. This helps us perform tasks in a cognitively less intensive fashion. Scripts store learned patterns of behaviour and outcome of different situations. For instance, paying for groceries follows a well defined script: stand in a line waiting for your turn, place the items on the belt, pay and go packing. By following this script, we do not have to figure out how to pay for our groceries every time we need to buy food. It is to be noted, though, that the scripts vary according to geographical and cultural areas. The script for visiting a grocery store or bank is different, e.g., in the USA, different parts of Europe or China.

A higher level theory of the same phenomenon is the one presented by Goffman (1959). According to his view, social life is assimilated to a theater play, where every participant is supposed to play their own role. In the level of interaction, the focus of his interest is in maintaining face. The common goal of the interlocutors in a conversa- tion is to maintain their own social face and those of the other participants.

\subsection{Usability and Design}

When we are dealing with dialogue systems, we cannot overlook the fact that we are inherently dealing with a user interface. In the fields of usability and design, the problem of communication has been dealt with from the human-computer interaction point of view.

A simple heuristic in usability for assessing a user interface is to look at the mental and physical effort (cf. Komogortsev et al., 2009) required to perform a task. For dialogue systems, physical effort can be calculated by how many queries the user has to perform to complete a given task. Mental effort refers to all cognitively demanding tasks such as how much information the user has to gather and memorize from different parts of the interface. Thus a dialogue system listing all the possible flights with all the details when requested would have low requirement for physical effort, but would be cognitively intensive as the user would have to memorize every flight he finds suitable.

Maybe a more intriguing concept in design is that of elegance (cf. White 2011). An elegant design communicates the intended message fully with as little as possible. The communication in a message can be divided in two: in denotation and connotation. Where denotation is the pure information content of the message, connotation is more in the way the message is communicated - in the emotional response it evokes.

\subsection{Synthesis}

In the previous sections, we have dedicated much room for describing the theories from different disciplines that in their core, are dealing with the very same phenomenon - communication. This section is dedicated into putting the theories together to form an interdisciplinary framework for a dialog system that is independent of the technical realization or creativity at this point.

We take elegance and script as higher level concepts as they are on the highest level of abstraction. Reflecting these in terms of the co-operative principle, i.e. the maxims, we can notice that elegance is closely related to the maxims of quantity and quality. As the requirement of elegance is to express the message as fully as possible (quality) 
with as little as possible (quantity), an elegant utterance needs to fulfill these two maxims.

Scripts are most strongly related to the maxims of manner and relevance. As scripts give us behavioral patterns to follow in different situations, they govern the manner in which we are expected to express ourselves. The behavioral patterns also entail what is relevant to say in which situation.

We place the usability terms on the lowest level in our synthesized model of co-operation, as they are meant to assess a concrete human-computer interaction scenario. Physical effort is linked mostly to the maxims of quantity and relevance. A dialogue system providing too little information will force the user to ask for more details, which increases the amount of physical effort. This is true also in the case of non-relevant information, which provokes more queries by the user to reach to a relevant answer.

The maxim of quantity relates to mental effort as well. Too much information will force the user to store it in his memory, which increases the mental effort. Another maxim affecting on mental effort is that of manner. If the information is not presented in an orderly manner, it makes it more difficult for the user to gather the important bits of information into a cohesive whole.

Coming back to the highest level concepts, elegance and script, a bridge needs to be built to connect them. We argue that they are connected through the context in which the conversation takes place. The context triggers a script, but it also changes the meaning of what is elegant. Talking with a person who knows a great deal about the topic of the conversation requires less words to communicate the message whereas more explaining is in order for a person new to the topic.

The context is also dictated by the role one is expected to play in the social situation. Therefore Goffman's theory is a part of the contextual bridge linking the two highest level concepts. We also introduce a mental model of the interlocutor as a part of the context as it has been proven evident by the previous discussion, that the maxims depend on the interlocutor as well. Furthermore, the conversation develops in time, which means that the prior utterances are also building the current context.

Now that we have synthesized what cooperative conversation requires, it is time to add the remaining notions into the model. No conversation can take place meaningfully if there is no message to be conveyed by the words of an utterance. This message can be divided into its denotative and connotative function. How the message can be conveyed is limited by the speech acts, and they function as a gate to the conversation.

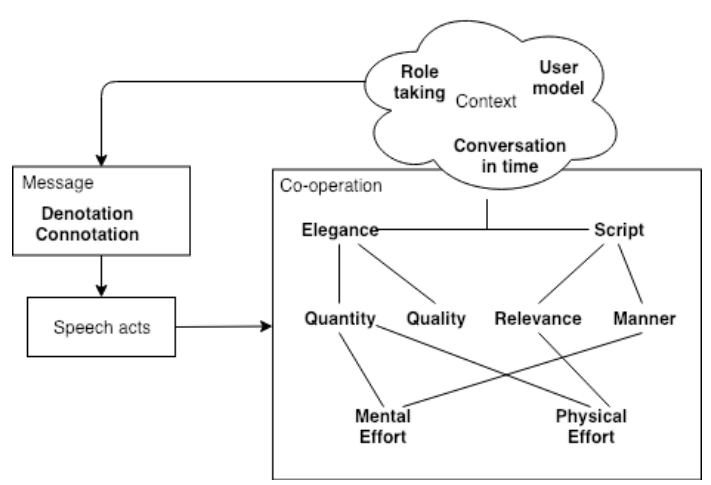

Figure 1: Model of co-operation

Figure 1 depicts the model described in this section. This model does not take creativity into account, but rather describes the requirements of cooperation and their inter-dependencies. The context is connected back into the message component as it affects on the next utterance of the conversation.

\section{Co-Operative Creativity}

In the previous section, we identified three main components of a co-operative dialogue system: message (including speech acts), context and cooperation, which correspond to message, contextual and communicative creativity respectively. In this section, we shed light into how computational creativity can manifest itself without jeopardizing the strict requirement of co-operation.

\subsection{Message Creativity}

In a co-operative setting, there is a limitation to what can be communicated so that it is still relevant for the conversation. The limitation can be very strict like in the case of a dialogue system selling tickets or lenient as in the case of chitchat.

\subsubsection{Creativity in Denotation}

Even if the set of possible denotations was limited, there is room for creativity in finding something else to communicate that is still co-operative. For example, glass is half full and glass is half empty communicate about the same phenomenon, yet their denotations are different. Thus, finding a creative point of view to communicate about the 
same phenomenon is a way of altering the denotation of the message without making it non-cooperative.

In a more lenient setting, the context of the message can be explored to find a way to communicate a denotation that still contributes to the cooperativity. This could, for instance, be a change of topic or a message provoking an emotional change such as a joke.

\subsubsection{Creativity in Connotation}

Even if the denotation of a message was fully fixed, for example, if the system has to communicate the price of a movie ticket and cannot communicate any other denotation to avoid risking cooperativity, connotation opens up more room for creativity.

Connotation can be altered as easily as by the choice of words or by a structural change. Consider for example the following sentences $A n$ appointment for vaccination has been reserved for Monday and You will get your shot on Monday. Both of them communicate the same denotation, but their connotation is different. The first sentence sounds more official and establishes social distance where as the latter is more casual in style.

\subsubsection{Exploiting Speech Acts}

Speech acts are more abstract in nature than any linguistic form, and thus speech acts themselves do not offer much room for creativity. However, understanding that certain surface realizations are most closely attached to certain speech acts, opens up a window for creativity.

I like strawberries is seemingly an expressive speech act; the person tries to communicate about his liking of strawberries. However, the actual speech act might be directive give me strawberries or commissive I will buy strawberries, depending on the context. Therefore mixing and matching speech acts with non-typical surface forms that still communicate the message is an exploitable possibility of computational creativity.

\subsection{Contextual Creativity}

The context has a huge effect on how communicated messages are understood. As we have seen throughout this paper, words can mean different things in different contexts. A context also sets limits to what can be said and how it should be said.

\subsubsection{User Adaptation}

Knowing the user and establishing trust with him gives more freedom for creative behavior. Even in tightly scripted situations, if the user is known well, the communication can deviate more from the script without it damaging the co-operation.

A semantic model that has been learned from the user in question could be used to creatively adapt a message to the user's own vocabulary. If for example the user hates frozen yogurt a flight connection with tediously long layovers could be communicated as a frozen yogurt route.

A creatively expressed message has a higher risk to not being understood. A good user model can then provide a way of assessing whether a creative communication solution will be understood as intended or not.

\subsubsection{Role Identification}

If we look at communication from the perspective of role-taking, a great source of creativity can come from identifying the possible roles supported by the context and picking the one that gives the greatest freedom in expression.

Role identification from user perspective, especially if there are many human users, can contribute to the creative freedom. If there are more than one possible roles the users can take, changing their role to one that offers more freedom of creativity can be of a benefit. The roles can be changed by communicative means.

\subsubsection{Time Perspective}

Planning the flow of the conversation ahead doing constant predictions is a potential way of shifting the context towards one that has more room for creativity. The planning itself can also be a creative process where the conversation will take unexpected turns that still contribute to co-operation.

Just as much as predicting the future can be a creative process, knowing the past can be used creatively as well. This is not limited to creative comebacks to what the user has said, but also can mean re-interpretation of what has been said before. Language is ambiguous and this fact can be celebrated by reusing bits of the conversation form the past in the new current context.

\subsection{Communicative Creativity}

The co-operation section in Figure 1 is probably the part that limits creativity the most. Maxims and the other components they relate to set rules 
to how one is supposed to communicate in order to do it in a co-operative fashion. However, there is room for creativity even with these tight rules.

\subsubsection{Script Selection}

In a conversation situation, there might be multiple social scripts to choose from. Picking a nontypical, but yet contextually fitting script can make it possible to find new creative solutions in the conversation.

As scripts are not predefined hand-written rules, but rather learned behavioral patterns, scripts offer flexibility in changing them. Identifying how to change a script, or how to go outside of one, in a way that it does not startle the interlocutor, is a task requiring creativity.

\subsubsection{Adjustment of Elegance}

Optimizing for elegance is probably too limiting for creativity and not an interesting way to go about creativity in conversation. The question should be what is elegant enough, and what is expected to be communicated. A longer message might be seen inelegant as it uses too many words to communicate a message, if we are only interested in the denotation. However, the additional length might contribute to connotation.

Making justified statements about elegance requires a definition of what is communicated, the message itself. This tells what is expected to be communicated, which then in its turn, makes it possible to assess the elegance of the utterance.

\subsubsection{Informed Deviation from Maxims}

Maxims are a part of co-operative principle and thus by definition they are tailored towards cooperative conversation. However, they are highly contextual and therefore what is enough, relevant and so on is a matter of the context in which an utterance occurs.

A system seeking to deviate from the maxims and still maintain co-operativity in the communication needs to be able to assess the effect of such a deviation in a reasoned way. For example, if the goal is to make the user think and ask questions, communicating a bit too little or increasing ambiguity might be useful.

A seemingly irrelevant communication can be useful if the communication is later contextualized and made relevant for the initial conversation topic. Sometimes telling anecdotes or giving analogous examples might seem irrelevant to the in- terlocutor, but later in the conversation they can prove to be helpful in understanding the problem from another perspective.

The maxim of quality relates to truthfulness of the utterance. Expressing something that is clearly untrue can be a way of expressing the opposite meaning in a sarcastic fashion (cf. Hämäläinen, 2016). If the sarcasm is understood correctly by the user, the communication can still be cooperative, even though on the surface it appears to be insincere.

\section{The Context of Peace Machine}

Peace Machine (Honkela, 2017) is a concept on how to use different parts of Artificial Intelligence (AI) to promote peaceful conditions in the world. This highly ambitious objective may sound unrealistic at first. It is to be remembered, though, that the range of AI technologies that have considerable impact in various domains is wide and increasing.

The Peace Machine concept consists of three main areas. The question is not about one system but a number of different applications and systems. The three main areas considered are (1) Improved communication, (2) Understanding emotions, and (3) Improving societal conditions.

\subsection{Co-Operative Creativity in Peace Machine}

In the following, the Peace Machine concept is considered from the point of view of Co-Operative Creativity defined and described in this paper. Peace Machine serves as a general application context for the theoretical work presented in this paper and its components can be studies in the communicative framework presented in this paper.

\subsection{Message Creativity}

The objective of Peace Machine is to help the user of a component of the system use and learn communicative acts that help him navigate in the conversational space in a peaceful and constructive manner or understand one's own or others' emotions in a constructive way. To be successful in this task, the system must be able to express itself in a creative manner when necessary. The user may need help in seeing matters from a novel point of view or in understanding the current situation beyond the limits of the conceptual system that he may have available. This help may be reached, for 
instance, with the use of metaphor.

\subsubsection{Creativity in Denotation}

The topic of conversation may be guided into areas in which, for instance, the risk of emotional outbursts are lowered. The creativity of the system would lie in the ability to guide the topics of the conversation even when the overall communicative goal remains the same. One opportunity is to find a path in the conversation that minimizes unintended choice of topics or expressions that might endanger the overall goal. It is known from practical experience in peace negotiations that the use of a poorly chosen single word or theme may jeopardize the whole process. Here it is to be remembered that Peace Machine is not focusing on peace negotiations between nations or other such organizations but between any two or more people.

\subsubsection{Creativity in Connotation}

In Peace Machine, consideration of the connotation is very important. When the aim is to reach peaceful and constructive communication, expressions that have negative connotations should be avoided. In a conversation between two people, the system may help the persons to avoid expressions that hurt other's emotions or the ground of his identity. In many cultures it is important to take social aspects into account. Depending on the relationship between the people, their status and cultural background, the expressions that are appropriate in one situation may be quite the opposite in another. For instance, the same content can be expressed in two quite different ways regarding the style: Let's have a meeting tomorrow! or May I have the honor to ask your presence in meeting in the near future, potentially already tomorrow?

\subsubsection{Exploiting Speech Acts}

Useful computational creativity that helps people through potentially problematic communication can take place through suitable choices regarding speech acts. In a homely context, there is a clear difference between the expressions Take out the trash bin and The trash bin is quite smelly. The intention can be considered to be the same in both cases but the emotional outcome may be quite different. Whether illocutionary, perlocutionary, propositional or utterance act should be chosen depends on multiple factors that concern the persons involved, their background, history of the communication and the broader context. At the present moment, it is still difficult to take into account the non-linguistic context in human-like manner. It is, however, good to keep in mind that persons may interpret the non-linguist or implicit context in a different way especially if they, for instance, have different education or cultural background (cf. Anderson and Shifrin, 2017).

\subsection{Contextual Creativity}

In Peace Machine, as in any general purpose system, the challenge of world knowledge and the huge complexity of the contexts that a system may encounter is a great challenge as well as an opportunity. This could be an indirect or direct access to the context. Here indirect refers to the use of language and the direct refers to use of perceptual senses. The underlying matters have been a subject to philosophical debates for very long time (cf. Gärdenfors, 2000; Von Foerster, 2007; Bundgaard, 2010) and it is not possible to cover this theme here. From the point of view of Peace Machine, the room for computational creativity is extensive and given broad range of opportunities. In building peace one possible approach is to choose the topics and dimensions suitably. For instance, the choice can help the discussants feel safe and secure. A useful notion is the division into foreground and background that is used in cognitive linguistics (Langacker, 2008). Sometimes it may be useful and constructive to start conversational from the background and gradually proceed into the foreground. The creative system may help humans in finding such conversational routes.

\subsubsection{User Adaptation}

In the above discussion referring to context, the aspect of subjectivity was briefly brought up. In addition to their experiences, values, preferences and identity, people are also different regarding their linguistic and conceptual systems. We do not know the same set of words and their meanings and we even have different interpretations of words and expressions. The words "fair" or "beautiful" refer to different things, which should be obvious, but more difficult to measure than comparing the limits or distributions of interpretation of color "orange" or whether some product is "expensive". In Peace Machine, this theme is very important as it has been pointed out that misunderstanding is a very common phenomenon that has wide practical consequences. Creative user adaptation on language and conceptual systems is pre- 
sented as a potentially important means to serve a basis for highly improved communication. This is a hypotheses that needs to be tested in various kinds of settings.

\subsubsection{Role Identification}

A machine, the purpose of which is to help people understand one another, can take up different roles in a communicative setting. In a situation of conflict, a suitable role might be that of a mediator while some situations require a more active leader-like role from the machine. This gives the machine a spectrum of roles from the passive to active to choose from.

\subsubsection{Time Perspective}

Helping people understand one another is a task with a persuasive goal. This persuasion requires planning, and the creative outcomes of the flow of the conversation have to be taken into account by the system.

With an aim for peace, Peace Machine should be able to take turns in the conversation that get the interlocutor off guard. In an extremely polarized setting, the two opposing parties are biased towards not being open towards the other party's opinions. A persuasion technique such as this one requires creative planning.

\subsection{Communicative Creativity for Peace Machine}

In the following, we consider how to communicate in a co-operative fashion while using the Peace Machine system.

\subsubsection{Script Selection}

Useful scripts to promote mutual understanding and respect can be learned based on large corpora of conversations. The real world variety of contexts makes its useful to apply creative solutions when the corpus-based solution does not provide close enough solution. Two or more solutions may be merged.

\subsubsection{Adjustment of Elegance}

Elegance is seemingly an important criterion regarding Peace Machine. The system should communicate in such a manner that it matches with the user's linguistic expectations and situationspecific needs. Too short and ambiguous message may be considered impolite or rude. Equally well, a message too long may be considered uninteresting or impolite. The Peace Machine system component can be used to train a person to handle potentially troublesome situations, during the conversation with someone else, or to help by analyzing an earlier conversation.

\subsubsection{Informed Deviation from Maxims}

From the point of view of the Peace Machine concept and system use, among the Grice's (1975) four maxims for co-operative principle of communication, manner, quality, quantity and relevance, can be used to judge potential usefulness of breaking these rules in a creative way. Regarding manner, the system may guide a person to be unclear or ambiguous in order to give room for alternative helpful interpretations or ideas, or to point out that the terminology and conceptual space may be such that meaning negotiation would be useful regarding the conversational situation at hand. The initial problem may help in understanding that the basis is not the same regarding the meaning of some key term in the conversation.

Changing the topic in the middle of a conversation and not being relevant may be a means to create a possibility to escape a problematic conversational situation. This approach should be used with care because it may lead into unintended consequences. For instance, the expression may be interpreted as an insult rather than as, for instance, humorous break to a heated discussion.

\section{Conclusions}

This paper has identified a need for theoretical framework for asymmetric human-computer creativity, where, for the first time, the computer is the only party with a requirement for creativity. Thus our initial framework fills a theoretical void in the field.

In this paper we have outlined from an interdisciplinary point of view what the requirements are for a co-operative conversation. Based on this definition, we have identified three different kinds of creativity in a co-operative setting: message, contextual and conversational creativity.

Furthermore, we have highlighted the importance of having a message to convey creatively. This makes a clear distinction with the creative systems that generate language without a need to communicate a certain idea, a message. Due to the nature of dialogue systems that are meant to aid users reach their goal, this need for a message cannot be ignored. 


\section{References}

Khalid Alnajjar and Mika Hämäläinen. 2018. A Master-Apprentice Approach to Automatic Creation of Culturally Satirical Movie Titles. In Proceedings of the 11th International Conference on Natural Language Generation (INLG), pages 274-283.

Richard C Anderson and Zohara Shifrin. 2017. The meaning of words in context. In Theoretical issues in reading comprehension, pages 331-348. Routledge.

Gordon H Bower, John B Black, and Terrence J Turner. 1979. Scripts in memory for text. Cognitive Psychology, 11(2):177 - 220.

Peer F Bundgaard. 2010. Husserl and language. In Handbook of phenomenology and cognitive science, pages 368-399. Springer.

Simon Colton. 2008. Creativity Versus the Perception of Creativity in Computational Systems. In AAAI Spring Symposium: Creative Intelligent Systems, Technical Report SS-08-03, pages 14-20, Stanford, California, USA.

Simon Colton, John William Charnley, and Alison Pease. 2011. Computational creativity theory: The face and idea descriptive models. In ICCC, pages 90-95.

Nicholas Davis. 2013. Human-computer co-creativity: Blending human and computational creativity. In Ninth Artificial Intelligence and Interactive Digital Entertainment Conference.

Erving Goffman. 1959. The Presentation of Self in Everyday Life. University of Edinburgh Social Sciences Research Centre.

H Paul Grice. 1975. Logic and conversation. 1975, pages $41-58$.

Peter Gärdenfors. 2000. Conceptual Spaces: The Geometry of Thought. MIT Press, Cambridge, MA, USA.

Mika Hämäläinen. 2016. Reconocimiento automático del sarcasmo - ¡Esto va a funcionar bien! Master's thesis, University of Helsinki, Finland. URN:NBN:fi:hulib-201606011945.

Timo Honkela. 2017. Rauhankone. Tekoälytutkijan testamentti. Gaudeamus.

Anna Jordanous. 2012. A standardised procedure for evaluating creative systems: Computational creativity evaluation based on what it is to be creative. Cognitive Computation, 4(3):246-279.

Pegah Karimi, Kazjon Grace, Mary Lou Maher, and Nicholas Davis. 2018. Evaluating creativity in computational co-creative systems. In The Proceedings of the Ninth International Conference on Computational Creativity, ICCC.
O Komogortsev, Carl J Mueller, Dan Tamir, and Liam Feldman. 2009. An effort based model of software usability. In 2009 International Conference on Software Engineering Theory and Practice (SETP-09).

Ronald Langacker. 2008. Cognitive grammar: A basic introduction. OUP USA.

Todd Lubart. 2005. How can computers be partners in the creative process: Classification and commentary on the special issue. International Journal of Human-Computer Studies, 63(4):365 - 369. Computer support for creativity.

Ikujiro Nonaka. 1994. A dynamic theory of organizational knowledge creation. Organization science, 5(1):14-37.

Vasile Rus, Cristian Moldovan, Nobal Niraula, and Arthur C Graesser. 2012. Automated discovery of speech act categories in educational games. International Educational Data Mining Society.

John Rogers Searle. 1969. Speech acts: An essay in the philosophy of language. Cambridge university press.

Heinz Von Foerster. 2007. Understanding understanding: Essays on cybernetics and cognition. Springer Science \& Business Media.

Alex W White. 2011. The Elements of Graphic Design. Allworth Press.

Geraint A Wiggins. 2006. A preliminary framework for description, analysis and comparison of creative systems. Knowledge-Based Systems, 19(7):449458.

Georgios N Yannakakis, Antonios Liapis, and Constantine Alexopoulos. 2014. Mixed-initiative cocreativity. In Proceedings of the 9th Conference on the Foundations of Digital Games. 